\title{
Factors associated with fatigue among truck drivers Literature review
}

\author{
Xinyi Ren ${ }^{1}$ \\ ${ }^{1}$ School of public health and preventative medicine, Monash university \\ Email:xren0009@student.monash.edu
}

\begin{abstract}
Fatigue is a significant dominating risk factor for transport workers, especially for long-distance truck drivers. Fatigued driving can easily lead to decreasing the drivers' judgment ability, slow reaction, and an increase of operational errors, and as well as increased probability of road traffic accidents. This study's aim is to identify the current evidence of risk factors contributing to fatigue for truck drivers. A literature search was made in 2021 and included articles back to the last ten years. All the factors can be divided into three aspects: demographics, workrelated and driver-related factors. The results showed that fatigue was closely related to travel-based payment, long shift, long-distance travel, and lack of sleep. However, conflicting results were found in terms of age and smoking. Therefore, more research is needed.
\end{abstract}

Keywords: fatigue, truck drivers, occupational health, road safety, risk factors

\section{INTRODUCTION}

Fatigue is defined as a subjective feeling of discomfort, and the loss of its ability to perform everyday activities or work under the same conditions.[1] Fatigue is common among professional drivers such as truck drivers. The nature of the occupation, irregular sleep patterns, long working hours and shift work increase the risk of insufficient sleep and fatigue.[2] Perceived pain or mental discomfort can also lead to increased fatigue while driving [3]. At present, fatigue driving has become the primary safety problem of the truck transportation industry. It can easily lead to decreased judgment ability, slow reaction and increase of operational errors, and as well as probability of road traffic accidents.[3] According to American federal statistics, truck drivers are more prone to drowsiness within 120, 000 national fatal and major traffic accidents than other drivers, with $13 \%$ of large truck crashes due to driving fatigue.[4] The high prevalence of fatigue is also reported in the UK drivers, where twothirds of drivers who fall asleep at the wheel are truck drivers, and about $40 \%$ of the sleep-related accidents involve commercial heavy vehicle drivers.[5]

In Australia, road transport has been the main form of freight transport for most commodities produced and consumed. It is essential to the national economy, which is estimated to occupy $14.5 \%$ of the GDP.[6] Across Australia, fatigue-related truck crashes are widely reported. According to the Transport Accident Commission, nearly one-fifth of fatal road accidents are concerned with the drivers being fatigued.[7] It is estimated that compared with drugs and alcohol, fatigue is four times more likely to cause work-related injuries, which means that fatigue is more harmful to road safety than drunk driving and speeding. [7] The Accident Research and Road Safety Center found that drowsiness, that is, extreme desire to fall asleep, causes nearly 20$30 \%$ of road deaths and serious injuries. [8] In addition, in a national fatigue-related car accident study, New South Wales had the highest traffic accident rate, accounting for $22 \%$, although $16.7 \%$ of fatal fatigue accidents occurred in Queensland. [9] Fatigue is also the leading cause of traffic accidents in Victoria, causing 50 deaths and nearly 300 serious injuries every year. [9]

\section{RATIONALE}

So far, the relationship between truck driver fatigue and fatigue-related behaviour has been widely discussed in the recognized literature. There are also many studies investigating work-related and non-work-related fatigue factors in other occupations, especially nurses. Long shifts (usually defined as $>10$ hours in 24 hours period), night shift working, intense practice environments and patients' dissatisfaction with their work contributed to increased fatigue levels. [10] Car drivers, bus drivers, taxi drivers, and various professional drivers also extensively discussed the impact and changes in driver 
behavior related to fatigue. [11] However, current research on truck drivers focuses more on the relationship between fatigue and road safety issues (such as crashes and violation). [12] There is limited research investigating what causes fatigue, or how and why risk factors influence fatigue among Australian truck drivers. In this case, this literature review aims to identify the current evidence of risk factors contributing to fatigue for truck drivers.

\section{METHODOLOGY}

This article conducts a literature review in four steps. First, a research question was raised: "What are the factors related to the fatigue driving of truck drivers?" Second, a literature search was conducted on two databases using the search terms formed by the research question (Table 1). Third, data extraction. Fourth, describe, analyze, and explain the existing evidence.

Table 1: Search terms used in literature search

\begin{tabular}{|l|l}
\hline Key Terms & Mesh Terms \\
\hline Truck drivers & $\begin{array}{l}\text { Professional driver, long-haul } \\
\text { driver, and truckers }\end{array}$ \\
\hline \multirow{2}{*}{ Fatigue } & $\begin{array}{l}\text { Muscle fatigue, physical fatigue, } \\
\text { mental fatigue, emotional fatigue, } \\
\text { tiredness, weariness, exhaustion, } \\
\text { drowsiness, and lethargy }\end{array}$ \\
\hline
\end{tabular}

A literature search was conducted using a systematic approach from two academic databases including PubMed and Ovid Medline. The terms "fatigue" and "truck driver" were used as key search terms. Muscle fatigue/ physical fatigue/ mental fatigue/ emotional fatigue/ tiredness/ weariness/ exhaustion/ drowsiness/lethargy were used as synonyms for fatigue. Truck driver/ professional driver/ long-haul driver/ truckers were used as synonyms for the truck driver to ensure detailed search results. After combining each mesh terms with the search function "OR", key terms were combined with "AND" and limiting search within the last ten years and English language only. The inclusion and exclusion criteria were as follows.

\subsection{Study Inclusion and Exclusion}

Studies will be included if:

- The main result is fatigue, lethargy or falls.

- They investigated the association between fatigue and truck drivers OR investigated the association between fatigue and different risk factors in truck drivers.

- Fatigue types are mental fatigue, physical fatigue, emotional fatigue, social fatigue, pain-related and chronic diseases.

- Truck drivers were included as study samples whether analyzing truck drivers independently or from a mix of professional drivers.

- The study designs were quantitative, including descriptive, correlational, cross-sectional study.

- They were published in the English language.

- They were published within ten years.

Studies will be excluded if:

- Fatigue was not the primary outcome.

- Sample populations were car drivers, bus drivers, train drivers, or taxi drivers only.

- They were grey literature, conference presentations or editorials.

\subsection{Search Outcomes}

As shown in Figure 1, after searching the two databases, a total of 519 articles from Medline $(n=297)$, PubMed $(n=222)$ were identified and imported into Endnote. A total of 79 duplications were removed by using the find duplicates function, with 440 articles remaining. Following the screening of titles and abstracts, 384 records were removed. Subsequently, 55 articles were eligible for full-text screening that met study inclusion. The final number of articles included in this review was eight studies. 


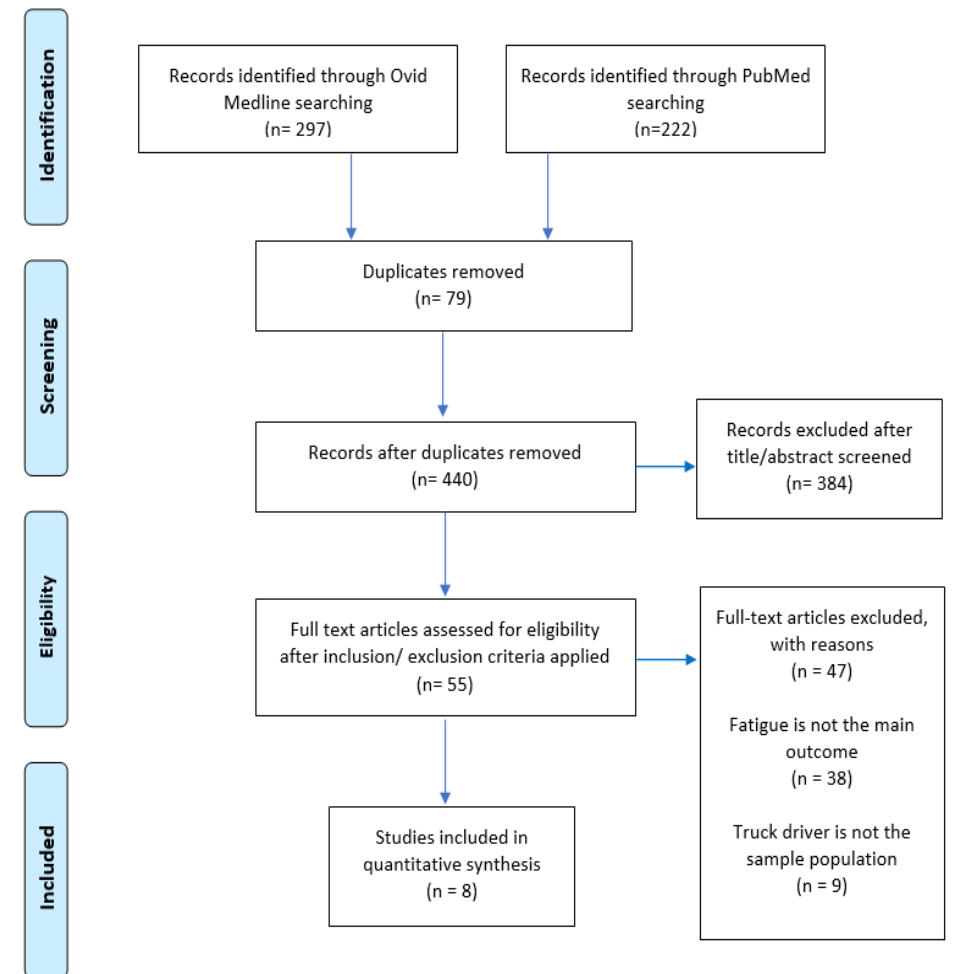

Figure 1: PRISMA diagram provides a graphical representation of the literature search ( $\mathrm{n}$ indicates number of studies included or excluded at each stage)

\subsection{Data extraction}

Data were extracted on the study characteristics included in the review. These included study design type, the country where the study was conducted, sample population with mean age, methods of collections, participants involved and summarized results. Risk factors were classified into demographic, work-related and driver-related factors.

\section{RESULTS}

\subsection{Literature features}

Of all eight articles, four were conducted in Europe, including Finland, Portugal, Italy, and Spain $[13,14,16,17]$. Two studies were conducted in Brazil $[18,19]$. One study was conducted in Australia. It is mainly conducted in the eastern coastal states of Australia. The other was carried out in Beijing, China $[15,20]$. All studies were cross-sectional research designs. Three studies $[16,17,20]$ used a combination of questionnaire surveys and interviews, and the other five studies only used questionnaire surveys $[13,14,15,17,19]$. A study conducted by Rosso et al. 28 used a Hard copy questionnaire. In addition, four studies focused on long-distance truck drivers $[13,16,18,20]$, three studies included a mixture of professional drivers such as taxi drivers and bus drivers $[14,15,17]$. One study focused only on Short-distance truck drivers [19].
The proportion of truck drivers in the mixed professional driver sample ranged from $48.9 \%$ to $71.6 \%$

\subsection{Literature results}

The risk factor associated with fatigue driving were organized into three categories: demographic factors, work-related factors including organizational and occupational factors, and driver-related factors.

\subsubsection{Demographic factors}

Age was the most common studied demographic factors. Rosso et al [14] stated that truck drivers who were over 55 years were five times more likely to fall asleep at the wheel and fatigue than those under 55 years old. However, another study conducted by TàpiaCaballero et al. [17] showed sleepiness was negatively correlated with age and openness to experience. A conflict result was found by a cross-sectional study from Perttula et al. [13], which showed gender, age and length of work experience were not statistically related to driver's fatigue.

\subsubsection{Work-related factors}

Work-related factors were most discussed in the literature, and the major work-related factors are organizational and occupational related factors. 


\subsubsection{Organizational factors}

Organizational factors refer to factors that affect the driver's workplace and work environment during the work process, including company size, rest time adjustment and workplace fatigue management. Large company size, no rest time, poor organization fatigue management, and long unpaid waiting time are related to high employee fatigue. $[13,15,20]$

\subsubsection{Occupational factors}

Occupational factors refer to occupational characteristics, work, and task level factors and can be attributed to three main characteristics: trip-based payment type, long work shift and long-distance trucking. Payment types were discussed in two studies. One founded that truck drivers were more likely to experience fatigue on half of trip if they received tripbased payment type $\left(\mathrm{X}^{2}=8.47\right)$ compared to time-based payment. [20] Another study found that compared with time-based payment methods, productivity-based payment methods are more prone to fatigue driving [18]. In addition, all studies have extensively discussed the shift system. Meng et al. [15] found that nearly half of the truck drivers reported that long-term driving is an essential factor in causing fatigue. Perttula et al. [13] reported that if driving for more than 16 hours, the risk of fatigue is 10 hours or less Quadruple. Also, compared to those who worked the day shift, the night shift was two times more likely at a higher risk of fatigue during work. [13] Furthermore, over 1,000 km long-distance trucking compared to $1,000 \mathrm{~km}$ or less, over 40000 miles (64 thousand kilometres) travelling per year than those less than that kilometer all showed statistically significant fatigue. [14, 18]

\section{DISCUSSION}

This study reviewed the factors associated with fatigue among truck drivers. Our findings indicated significant association between fatigue and demographic, work-related and driver-related factors.

Age is established as a predictor of sleepiness and fatigue. Most studies identified that advanced age (drivers aged 55 years or above) had an increased risk of fatigue and falling asleep at the wheel compared to younger drivers. [14] This could be explained by attention and reaction time decreasing along with age which leads to fatigue driving and poor drivers' performance. [21] However, it is well known that aging hurts driver performance. Young drivers are susceptible to insufficient sleep time and poor sleep habits [17]. This is consistent with the research of Philips et al. [22]. The reaction time of young drivers (20-25 years old) after insufficient sleep before work is affected. More extensive, and the reaction time of older drivers are not affected (52-63 years old). In addition, compared with experienced drivers, novice drivers were slow to perceive danger at first and lack of sleep can seriously impair their ability to predict traffic hazards. [23] There is a U-shaped relationship between age and risk. Therefore, further research is required regarding the relationship between age, reaction speed and fatigue, and the causes of insufficient sleep among young drivers due to the existing conflict.

Even though drivers can manage part of their fatigue, work-related strategies are essential. Truck drivers who were unable to choose the timing of their rest and break time would experience more fatigue during driving and easier to get involved in crash and traffic accidents than those who were constantly being able to. [13] There was also a high percentage of self-reported fatigue among truck drivers in large companies compared to small companies. [13] Besides, lacking fatigue management in the workplace and poor organizational safety culture might be in a position to influence how drivers behave at work and, in turn, cause fatigue experience. [15] Current evidence about fatigue management is about protection against human errors and technical failure and protection against the road accident. There is still a need to exploring more on fatigue management strategies.

According to Perttula et al. [13], it stated those working extremely long hours (more than 16 hours in 24 hours occupied a more significant proportion of driving fatigue. Williamson and Friswell [20] also concluded that long work shifts increased the driver fatigue level, especially for those with little waiting or queuing time on their trip. This often forced an increase in their workload and thus longer work hours. The additional work hours were generally not accounted for as part of the driver's salary packages, meaning the drivers were not paid for the waiting or overtime work.

In addition, long-distance truck transportation is highly related to fatigue and falling asleep on the steering wheel, especially when driving more than 64,000 kilometres per year [14,18]. Evidence shows that long shifts are related to short sleep time or sleep disorders, especially those over 8 hours. Shifts [24] The decrease in neurocognitive function and performance due to sleep deprivation may lead to fatigue, decreased alertness, and slower reaction time [22]. Other studies have confirmed physiological drowsiness, such as train drivers in Crew working at night and on long-distance flights.[25]

Work shift, distance travelled, and payment type was often interconnected. Truck drivers were more likely to experience fatigue if they received trip-based payment type. [20] The essence of this payment type is based on kilometres travelled, or tonnage and quantity carried. [20] Sleep-deprived before departure, long work hours, and long-distance trucking are usually associated with trip-rate payment type. Similarly, Girotto et al. [18] 
found that formal employment with productivity-based payment was closely related to driving fatigue and sleepiness. However, an obvious limitation of the pieces of literatures we found was the comparatively low participation rate to questionnaires. This may induce response bias, and further research is needed to address that.

In dealing with fatigue, one cannot admit that one of the most common and apparent causes of work fatigue is sleep deprivation. Our findings suggested fatigue driving was more common when drivers had insufficient sleep 10 hours before the last trip. [20] This is similar to Catarino et al. [16], who found sleep deprivation, significantly less than 8 hours per night was associated with a higher risk of drivers' sleepiness and excessive somnolence. Based on current standard guidelines in Australia, in 24 hours, the driver's working time must not exceed 12 hours and must have a continuous fixed time of at least 7 hours. [26] Thus, short sleep over, 24 hours and insufficient sleep conditions emerged as a factor negatively influencing the high frequency of fatigue. Sleep deprivation will reduce the driver's alertness, affect coordination, judgment, and reaction time, leading to fatigue driving and road traffic accidents [22]. Therefore, lack of sleep is the most likely place for other factors to have a significant impact. For example, irregular working hours and long-distance travel can lead to poor sleep conditions, leading to fatigue.

Diet and smoking were the most discussed lifestyle behaviors contributing to fatigue. Interestingly, diet and unhealthy meal consumption with high glycemic index, high sugar and rich in fats produced high sleepiness in short-haul drivers during the day. [19] Nevertheless, a contradictory study shows that eating habits have nothing to do with fatigue.[14] Thus, the effect of dietary habits on fatigue required further investigation. Furthermore, current evidence shows that smoking is associated with daytime sleepiness, especially smoking more than 20 cigarettes a day.[16] Although smoking is a standard self-report method for truck drivers to wake up when feeling fatigued, this lifestyle behavior may be related to excessive daytime sleepiness and may cause further fatigue of the driver.[14] Moreover, studies had shown that smokers had lower lung capacity than nonsmokers, and reduced oxygen in the lungs, brain, and body systems could exacerbate fatigue. [27] On the other hand, nicotine in cigarettes is a stimulant and disrupts sleep, which in turn increases the risk of sleep conditions such as sleep apnea. [27] Given the little evidence available on this topic, it remains to be seen whether smoking as a means of self-managing fatigue aggravates or mitigates fatigue.

\section{CONCLUSION}

This literature review analyzed eight works of literature on the factors of truck drivers' fatigue. They have established a comprehensive risk model of individual characteristics, organizational factors, occupational factors, lifestyle behaviors and other personal factors. Travel-based payment methods, long shifts, and long-distance trucking affect each other and become the main work-related factors that cause driver fatigue. Fatigue caused by sleep deprivation is the most discussed because it reduces coordination, alertness, and reaction times. In addition, the relationship between age, responsiveness, and fatigue, as well as the effects of smoking, needs further research. To a certain extent, this will promote the public's health awareness of driving fatigue, increase the awareness of drivers, and lay the foundation for the formulation and improvement of fatigue management regulations. This will also help shape the company's employee training program, formulate more realistic salary standards and more effective regulatory requirements. The assessment results will also accumulate knowledge for further research on how these risk factors affect fatigue.

\section{REFERENCES}

[1] Safe Work Australia. Fatigue. [Internet]. Canberra ACT: Safe Work Australia; [cited 2021 Mar 24]. Available from: https://www.safeworkaustralia.gov.au/fatigue

[2] Williamson AM, Feyer AM, Friswell R. The impact of work practices on fatigue in long distance truck drivers. Accident Analysis \& Prevention. 1996 Nov 1;28(6):709-19.

[3] Gastaldi M, Rossi R, Gecchele G. Effects of driver task-related fatigue on driving performance. Procedia-Social and Behavioural Sciences. 2014 Feb 5; 111:955-64.

[4] Federal Motor Carrier Safety Administration (FMCSA) [Internet]. Washington D.C.: FMSCA; [updated 2007 Jul; cited 2021 Mar 31]. Available from: https://www.fmcsa.dot.gov/safety/researchand-analysis/large-truck-crash-causation-studyanalysis-brief

[5] The Royal Society for the Prevention of Accidents (ROSPA). Driver Fatigue and Road Accidents Factsheet [Internet]. Birmingham: ROSPA; [updated 2020; cited 2021 Apr 3]. Available from: https://www.rospa.com/media/documents/roadsafety/driver-fatigue-factsheet.pdf

[6] Infrastructure Australia. Australia's growing freight task: Challenges and opportunities. [Internet]. Canberra ACT: Infrastructure Australia; [updated 2018 Oct 31; cited 2021 Apr 4]. Available from: 
https://www.infrastructureaustralia.gov.au/listing/s peech/australias-growing-freight-task-challengesand-opportunities

[7] Transport Accident Commission (TAC). Fatigue statistics. [Internet]. Victoria VIC: TAC; [updated 2000; cited 2021 Mar 31]. Available from: https://www.tac.vic.gov.au/road-

safety/statistics/summaries/fatigue-statistics

[8] Centre for Accident Research \& Road Safety Queensland (CARRS-Q). Sleeping and fatigue. [Internet]. Queensland: CARRS-Q; [updated 2015 Jul; cited 2021 Apr 8]. Available from: https://research.qut.edu.au/carrsq/wpcontent/uploads/sites/45/2017/04/Sleepiness-andfatigue-SCREEN.pdf

[9] Dobbie K. Fatigue-related crashes: An analysis of fatigue-related crashes on Australian roads using an operational definition of fatigue. 2002 May.

[10] Alahmadi BA, Alharbi MF. Work-related fatigue factors among hospital nurses: An integrative literature review. Nurse Media J. N. 2018; 8:11333.

[11] Biggs H, Dingsdag D, Stenson N. Fatigue factors affecting metropolitan bus drivers: A qualitative investigation. Work. 2009 Jan 1;32(1):5-10.

[12] Mahajan K, Velaga NR, Kumar A, Choudhary P. Effects of driver sleepiness and fatigue on violations among truck drivers in India. International journal of injury control and safety promotion. 2019 Oct 2;26(4):412-22.

[13] Perttula P, Ojala T, Kuosma E. Factors in the fatigue of heavy vehicle drivers. Psychological Reports. 2011;108(2):507-14.

[14] Rosso GL, Perotto M, Feola M, Caramella M. Falling asleep at the wheel among Italian professional drivers (PDs): results from the HiRis PD study. International Journal of Occupational Medicine \& Environmental Health. 2014; 27(6):1005-12.

[15] Meng F, Li S, Cao L, Li M, Peng Q, Wang C, et al. Driving fatigue in professional drivers: a survey of truck and taxi drivers. Traffic Injury Prevention. $2015 ; 16(5): 474-83$.

[16] Catarino R, Spratley J, Catarino I, Lunet N, PaisClemente M. Sleepiness and sleep-disordered breathing in truck drivers: risk analysis of road accidents. Sleep Breath. 2014; 18(1):59-68.

[17] Tàpia-Caballero $\mathrm{P}, \quad$ Serrano-Fernández $\mathrm{MJP}$, Boada-Cuerva M, Sora B, Boada-Grau JP. Influence that job characteristics, personality, and burnout have on fatigue in professional drivers. Int J Occup Saf Ergon. 2021:1-29.

[18] Girotto E, Bortoletto MSS, González AD, Mesas AE, Peixe TS, Guidoni CM, et al. Working conditions and sleepiness while driving among truck drivers. Traffic Inj Prev. 2019;20(5):504-9.

[19] Martins AJ, Martini LA, Moreno CRC. Prudent diet is associated with low sleepiness among shorthaul truck drivers. Nutrition. 2019; 63-64:61-8.

[20] Williamson A, Friswell R. The effect of external non-driving factors, payment type and waiting and queuing on fatigue in long distance trucking. Accident Analysis \& Prevention. 2013; 58:26-34.

[21] Bunce D, Young MS, Blane A, Khugputh P. Age and inconsistency in driving performance. Accident Analysis \& Prevention. 2012 Nov 1; 49:293-9.

[22] Philip P, Taillard J, Sagaspe P, Valtat C, SanchezOrtuno M, Moore N, Charles A, Bioulac B. Age, performance and sleep deprivation. Journal of sleep research. 2004 Jun;13(2):105-10.

[23] Philip P, Taillard J, Quera-Salva MA, Bioulac B, Åkerstedt T. Simple reaction time, duration of driving and sleep deprivation in young versus old automobile drivers. Journal of Sleep research. 1999 Mar;8(1):9-14.

[24] Cheng WJ, Cheng Y. Night shift and rotating shift in association with sleep problems, burnout and minor mental disorder in male and female employees. Occupational and environmental medicine. $2017 \mathrm{Jul}$ 1;74(7):483-8.

[25] Akerstedt T, Wright KP. Sleep loss and fatigue in shift work and shift work disorder. Sleep medicine clinics. 2009 Jun 1;4(2):257-71

[26] National Heavy Vehicle Regulator (NHVR). Standard hours. [Internet]. Canberra ACT: NHVR; [cited 2021 Apr 18]. Available from: https://www.nhvr.gov.au/safety-accreditationcompliance/fatigue-management/work-and-restrequirements/standard-hours

[27] Corwin EJ, Klein LC, Rickelman K. Predictors of fatigue in healthy young adults: moderating effects of cigarette smoking and gender. Biological research for nursing. $2002 \mathrm{Apr}$; 3(4): 222-33. 